\title{
ADESÃO À TERAPIA ANTIRRETROVIRAL PARA HIV/AIDS
}

Stela Maris de Mello Padoin 1 , Samuel Spiegelberg Zuge², Érika Eberlline Pacheco dos Santos ${ }^{3}$, Marcelo Ribeiro Primeira ${ }^{3}$, Juliane Dias Aldrighi ${ }^{3}$, Cristiane Cardoso de Paula ${ }^{4}$

RESUMO: O objetivo do estudo foi determinar a adesão à Terapia antirretroviral de adultos com Vírus da Imunodeficiência Humana/Síndrome da Imunodeficiência Adquirida atendidos em um hospital universitário do Sul do Brasil. É uma pesquisa de abordagem quantitativa, descritiva com delineamento transversal; realizada em 2011. Os dados foram analisados com o auxílio do aplicativo SPSS $®$ (Versão 17.0). A população do estudo foi de 125 pacientes, na faixa etária de 20 a 49 anos; 44,0\% foram classificados como não aderentes, destes, 29,3\% deixaram de tomar os medicamentos por uso de álcool ou drogas; $26,2 \%$ devido aos efeitos colaterais; $17,6 \%$ por motivos diversos e $4,5 \%$ por estarem trabalhando. Destaca-se a necessidade de avaliar o comportamento a fim de realizar ações educativas que permitam cooperação dos pacientes para maior adesão do tratamento, reduzindo a morbidade e mortalidade desse agravo à saúde.

DESCRITORES: Síndrome da imunodeficiência adquirida; HIV; Terapia antirretroviral de alta atividade; Adesão à medicação; Enfermagem.

\section{ADHERENCE TO ANTIRETROVIRAL THERAPY FOR HIV/AIDS}

ABSTRACT: The study's objective was to determine the adherence to antiretroviral therapy in adults with the Human Immunodeficiency Virus/Acquired Immunodeficiency Syndrome, attended in a university hospital in the south of Brazil. This research has a quantitative approach, and is descriptive with a cross-sectional design, and was undertaken in 2011. The data was analyzed using the SPSS ${ }^{\circ}$ program (version 17.0). The study population was 125 patients, in the age range of 20 to 49 years old; $44.0 \%$ were classified as non-adherent, of whom $29.3 \%$ stopped taking the medications because of use of alcohol or drugs; $26.2 \%$ due to the side effects; $17.6 \%$ for various reasons, and $4.5 \%$ because they were working. The need is emphasized to assess the behavior so as to undertake educational actions which allow the patients' cooperation for greater adherence to the treatment, reducing the morbidity and mortality of this health issue.

DESCRIPTORS: Acquired Immunodeficiency Syndrome; HIV; High activity antiretroviral therapy; Adherence to the medication; Nursing.

\section{ADHESIÓN A LA TERAPIA ANTIRRETROVIRAL PARA HIV/AIDS}

RESUMEN: El objetivo de este estudio fue determinar la adhesión a la Terapia antirretroviral de adultos con Virus de la Inmunodeficiencia Humana/Síndrome de la Inmunodeficiencia Adquirida atendidos en hospital universitario del Sur de Brasil. Es una investigación de abordaje cuantitativo, descriptivo con delineación transversal; realizada en 2011. Los datos fueron analizados con la ayuda del aplicativo SPSS ${ }^{\circledR}$ (Versão 17.0). La población del estudio fue de 125 pacientes, en la franja etaria de 20 a 49 años; 44,0\% fueron clasificados como no adherentes, de estos, 29,3\% pararon de tomar los medicamentos por uso de alcohol o drogas; $26,2 \%$ a causa de los efectos secundarios; $17,6 \%$ por motivos diversos y 4,5\% por estar trabajando. Se destaca la necesidad de evaluar el comportamiento para realizar acciones educativas que permitan cooperación de los pacientes para mayor adhesión del tratamiento, reduciendo la morbidez y mortalidad de ese agravio a la salud. DESCRIPTORES: Síndrome de la inmunodeficiencia adquirida; HIV; Terapia antirretroviral de alta actividad; Adhesión a la medicación; Enfermería.

\footnotetext{
*Este artigo deriva da pesquisa intitulada "Indicadores de vulnerabilidade de adultos e idosos na adesão ao tratamento antirretroviral em serviços de referência da metade sul do Rio Grande do Sul", apoiada por meio de Programas de Bolsas de Iniciação Científica da Universidade Federal de Santa Maria - UFSM, do CNPq e da Fundação de Amparo à Pesquisa do Estado do Rio Grande do Sul- FAPERGS.

${ }^{1}$ Enfermeira. Doutora em Enfermagem. Professora do Departamento e do Programa de Pós-Graduação em Enfermagem - PPGENF da UFSM. Coordenadora do PPGENF UFSM. Líder do Grupo de Pesquisa Cuidado à Saúde das Pessoas, Famílias e Sociedade - GP PEFAS. ${ }^{2}$ Enfermeiro. Mestre em Enfermagem. Membro do GP PEFAS.

${ }^{3}$ Enfermeira. Membro do GP PEFAS.

${ }^{4}$ Enfermeira. Doutora emEnfermagem. Professorae Vice-coordenadora doCurso de Graduação emEnfermagem daUFSM.Líderdo GPPEFAS.
} 


\section{INTRODUÇÃO}

A partir do surgimento do Vírus da Imunodeficiência Humana (HIV) e da notificação de casos de adoecimento pela Síndrome da Imunodeficiência Adquirida (Aids), o Ministério da Saúde do Brasil utiliza-se de políticas públicas com a finalidade de prevenir novos casos da infecção e melhorar a atenção em saúde para as pessoas que convivem com a doença. Dentre elas, destaca-se a política nacional de distribuição gratuita dos medicamentos que compõe a Terapia Antirretroviral (TARV) para qualquer cidadão infectado pelo HIV e/ou com Aids. Esta política implicou na redução da morbidade e mortalidade dessas pessoas, reduziu o número de internações e aumentou sua expectativa de $\operatorname{vida}^{(1-2)}$.

Dessa forma, é possível considerar a adesão à TARV como o maior determinante para o sucesso terapêutico, pois há influência significativa nas condições clínicas e biológicas dos pacientes com HIV/Aids. A taxa de adesão exigida/esperada, quando se trata da TARV, é de $100 \%$, ou seja, a total conformidade entre a ingesta dos medicamentos e a prescrição médica, sendo que o paciente necessita entender e concordar com a prescrição recomendada ${ }^{(3-4)}$.

A adesão faz parte de um processo, o qual está intrinsecamente articulado às adequações cotidianas e ao comportamento das pessoas com HIV/Aids ${ }^{(3,5)}$. Porém, essas adequações ao tratamento dependem da disponibilidade de acesso à terapia, do acompanhamento clínico, da retirada dos medicamentos, tornando assim, a adesão à TARV um processo complexo, interativo, dinâmico e contínuo ${ }^{(6)}$.

Neste contexto, o presente estudo teve como objetivo determinar a adesão à Terapia Antirretroviral de adultos com HIV/Aids, atendidos em um Hospital Universitário do Sul do Brasil, a partir de variáveis comportamentais.

\section{MÉTODO}

Esta pesquisa apresenta uma abordagem quantitativa, do tipo descritiva e de delineamento transversal. Os critérios de inclusão foram dos participantes foram: adultos na faixa etária de 20 a 49 anos, de ambos os sexos, que realizavam acompanhamento no Ambulatório de Doenças Infecciosas do Hospital Universitário de Santa Maria, e que retirassem medicamento antirretroviral há no mínimo três meses. Foram excluídos os adultos que apresentassem alguma limitação a qual dificultasse a compreensão e expres- são verbal. Para a coleta de dados foi realizada uma entrevista estruturada, no período de julho a dezembro de 2011, sendo os pacientes selecionados a partir da chegada para o atendimento no referido ambulatório.

Foi criado um banco de dados no software Epi Info versão 7.0, com digitação dupla e independente e realizada análise descritiva das características sociodemográficas, econômicas e comportamentais, e análise das variáveis comportamentais de classificação da adesão à TARV, no software Statistical Package for Social Science, versão 17.0.

Foram definidas as variáveis comportamentais de classificação, e determinação de pacientes como aderentes ou não aderentes. Foi adotado como aderente o paciente que relatou ingestão de $100 \%$ das doses prescritas ${ }^{(4)}$.

Os aspectos éticos do estudo foram respeitados, seguindo à Resolução 196/96, sendo aprovado no Comitê de Ética em Pesquisa da UFSM, sob o número 23081.008437/2007-19.

\section{RESULTADOS}

A população do estudo foi composta por 125 pacientes em TARV para o HIV/Aids.

As características sociodemográficas e econômicas mostraram que $47,2 \%$ dos entrevistados estavam na faixa etária de 30 a 39 anos, $51,2 \%$ do sexo masculino; $85,5 \%$ solteiros e $14,5 \%$ casados. Quanto à raça, $64,8 \%$ autodefiniram-se como de cor ou raça branca, $30,4 \%$ como nego, $3,2 \%$ como indígena e $1,6 \%$ com de cor ou raça amarela. Relativo ao ensino, $49,6 \%$ possuíam o Ensino Fundamental Incompleto, 51,2\% trabalhavam fora de casa, $59,2 \%$ possuíam renda fixa e $49,6 \%$ tinham renda mensal da família menor que dois salários mínimos, sendo que 51,2\% trabalhavam fora de casa (Tabela 1).

Quanto às características comportamentais desses pacientes, destaca-se que em $76,8 \%$ a categoria de exposição ao HIV foi a sexual, $58,4 \%$ faziam uso de bebidas alcoólicas, destes, $38,5 \%$ relataram que beberam, mas não ficaram "altos" na última semana e 89,6\% não utilizaram drogas ilícitas na última semana (Tabela 1).

Segundo as variáveis comportamentais classificatórias de adesão, definiu-se que $44,0 \%$ dos adultos foram classificados como não aderentes, destes, $17,6 \%$ deixaram de tomar alguma dose dos ARV por algum motivo; $29,3 \%$ dos adultos que faziam uso de álcool ou drogas deixaram de tomar os ARV por esse motivo; $26,2 \%$ relataram que os efeitos colaterais os impediram de tomar os ARV; $4,5 \%$ deixaram de tomar por estarem no horário de trabalho (Tabela 2). 
Tabela 1 - Características sociodemográficas, econômicas e comportamentais dos adultos em Terapia antirretrovial para o HIV/Aids. Santa Maria, 2011

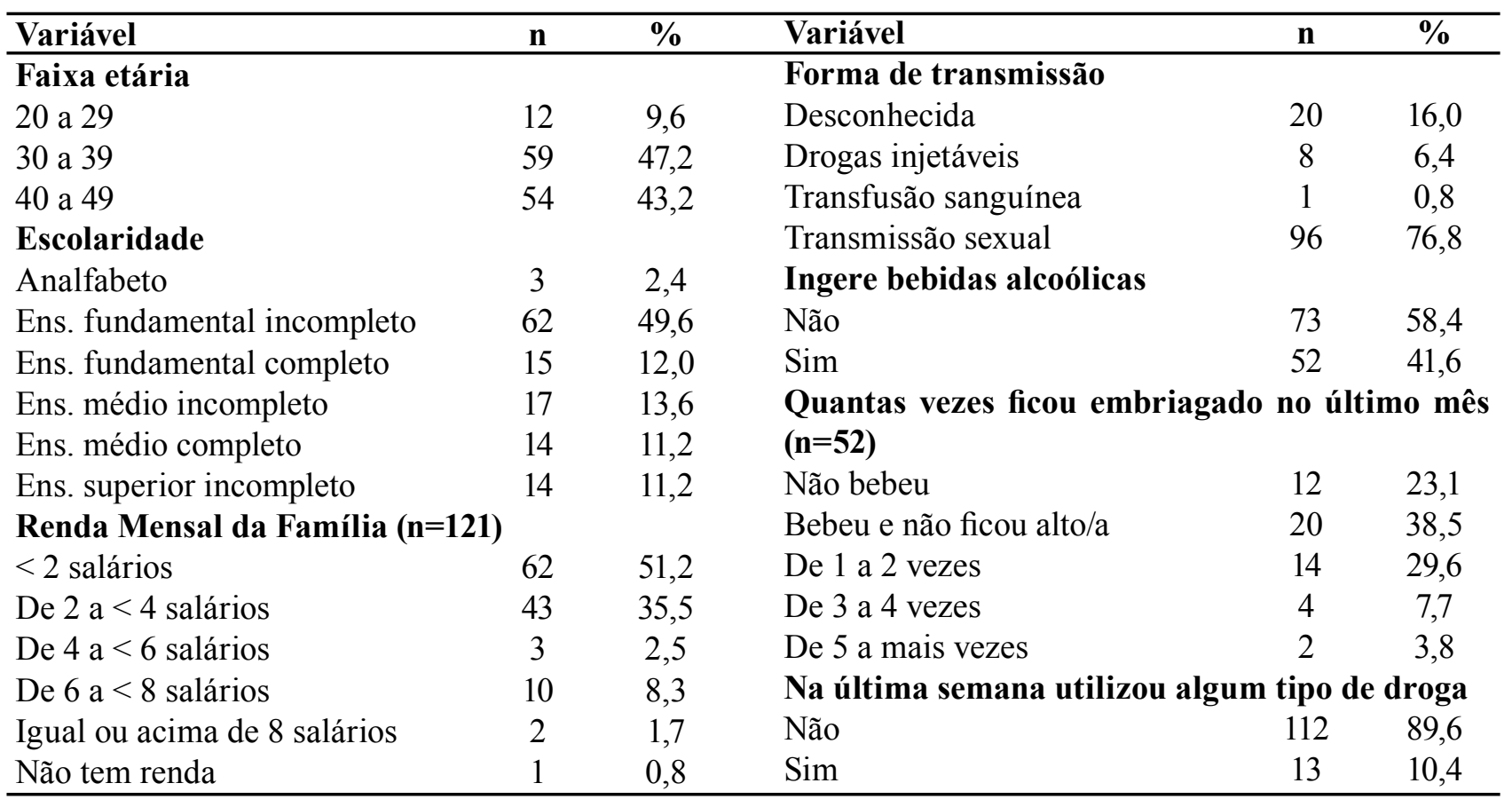

Tabela 2 - Variáveis de classificação da adesão à Terapia antirretroviral de adultos com HIV/Aids. Santa Maria, 2011

\begin{tabular}{lcc}
\hline Variável & $\mathbf{n}$ & $\mathbf{\%}$ \\
\hline Na semana passada, anteontem ou ontem deixou de tomar alguma dose do medicamento $(\mathbf{n = 1 2 5})$ \\
Não & 103 & 82,4 \\
Sim & 22 & 17,6 \\
Deixou de tomar os medicamentos por uso de álcool ou drogas & $(\mathbf{n}=\mathbf{1 2 3})$ & \\
Não & 36 & 70,7 \\
Sim & 87 & 29,3 \\
O efeito colateral impediu de tomar algumas doses do medicamento $(\mathbf{n = 4 2})$ & 73,8 \\
Não & 31 & 26,2 \\
Sim & 11 & \\
Você toma essas doses no horário de trabalho $(\mathbf{n}=\mathbf{2 2})$ & 1 & 4,5 \\
Não & 21 & 95,5 \\
Sim & & \\
\hline
\end{tabular}

\section{DISCUSSÃO}

A epidemia do HIV/Aids já completou três décadas e se perpetua como um problema de saúde pública devido à atual condição de cronicidade, ao seu caráter epidemiológico $^{(7)}$ e às condições de vulnerabilidade que atingem milhares de pessoas ${ }^{(4)}$, principalmente na faixa etária dos 30 a 39 anos e de cor ou raça branca ${ }^{(8)}$.

Observa-se que, embora a maioria dos pacientes da pesquisa sejam do sexo masculino, a razão homem/mulher neste estudo se aproxima de 1:1, com baixa escolaridade e condições econômicas precárias, convergindo com o processo de feminização e pauperização da epidemia no país ${ }^{(9)}$.
As questões relacionadas à feminização da Aids são caracterizadas, geralmente, pela ideia de invulnerabilidade das mulheres, por viverem em um relacionamento estável e pelas dificuldades que elas encontram ao negociar o uso do preservativo com o parceiro. Este fato remete à submissão feminina, consequência de relações desiguais de gênero e poder que, historicamente, retratam uma mulher oprimida, subjugada, e com poder limitado no campo das relações afetivo-sexuais. Por esse motivo, consciente ou inconscientemente, em algum momento do relacionamento, essas mulheres submetem-se às escolhas de seus parceiros tornando-as cada vez mais vulneráveis à epidemia ${ }^{(10-11)}$. 
Além disso, o grau de escolaridade tem se apresentado como um fator importante para o sucesso do tratamento, pois quanto maior a escolarização, maior é a compreensão de informações referentes ao HIV e aos cuidados de saúde. Pacientes com ensino fundamental incompleto apresentam grande probabilidade de não realizar a terapia adequadamente em comparação aos que possuem o ensino fundamental completo ${ }^{(12)}$.

O baixo grau de escolaridade, associado ao baixo poder aquisitivo, interferem diretamente na realização da TARV ${ }^{(12)}$, pois o elevado número de pessoas que não trabalha relacionado às características sociodemográficas e econômicas, apresentadas nesse estudo, caracterizou uma população que vive em condições precárias ${ }^{(13)}$.

Os resultados deste estudo apontam a prevalência de pessoas solteiras, condição civil que pode torná-los mais vulneráveis a não realizar a TARV, conforme estudos que associam a falta de suporte social com o abandono da TARV ${ }^{(14)}$. Porém, a razão de manter-se solteiro, pode ser pelo fato de que os pacientes, a fim de evitar possíveis problemas afetivos por causa das condições que a doença acaba causando, evitam se relacionar com outras pessoas preferindo o isolamento social ${ }^{(9)}$.

Relacionado às questões comportamentais dos pacientes em TARV, destaca-se que a forma de transmissão do HIV prevalece sendo a sexual, convergindo com os dados notificados na faixa etária do estudo ${ }^{(8)}$. Essa forma de transmissão ainda é a mais comum, pois o receio de infectar-se por alguma Doença Sexualmente Transmissível, inclusive a Aids, é muito pequeno, e quando o indivíduo percebe-se em uma situação de risco, as estratégias de prevenção não conduzem ao uso do preservativo, mas sim à seleção de parceiros, alterações comportamentais e questionamentos a respeito do comportamento passado desse parceiro ${ }^{(15)}$.

O álcool e o uso de outras drogas remetem ao comportamento do paciente frente à doença, podendo ser um fator prejudicial para a realização da TARV, contribuindo para menor sobrevida dos pacientes com HIV/Aids ${ }^{(16)}$. Porém, alguns casos não apresentam associação entre a realização correta da TARV e o uso de outras drogas consideradas recreativas, inclusive $o$ álcool ${ }^{(17)}$, devendo assim, avaliar as condições clínicas e biológicas individuais dos pacientes, a fim de perceber se o uso de álcool ou droga está, realmente, interferindo na eficácia da TARV.

A adesão é permeada por questões que envolvem a superação das dificuldades, mudanças comportamentais e de estilo de vida ${ }^{(18)}$. A classificação de adesão à
TARV é baseada nas características comportamentais dos pacientes em tomar ou não a medicação. Assim, os achados desse estudo - $44 \%$ de não aderentes - não convergem como os dados encontrados na literatura, onde a não adesão varia em torno de 20 a $30 \%{ }^{(19)}$. No entanto, destaca-se que as características de avaliação podem variar, dependendo do tipo de medida, do desenho do estudo e do ponto de corte da adesão ${ }^{(20)}$.

Os pacientes relataram que o principal motivo para não terem tomado a medicação foi o esquecimento. Essa irregularidade nos horários, e o não cumprimento da prescrição médica, podem estar associados a não aceitação do seu diagnóstico e às atividades cotidianas e de lazer ${ }^{(4)}$.

O fato de deixar de tomar os medicamentos por uso de álcool ou drogas é um fator que pode prejudicar a realização do tratamento. Há tendência de redução na frequência do uso desses à medida que aumenta a gravidade de transtornos relacionados ao uso de álcool ou outras drogas. Mesmo não havendo o consumo diário, existe, ainda, um consenso errôneo de que as pessoas devam deixar de tomar os medicamentos ao utilizar drogas ${ }^{(19,21)}$.

A utilização diária dos medicamentos $\mathrm{ARV}$ pode causar reação adversa e/ou causar um efeito indesejável nos pacientes, uma vez que, dependendo do tipo e frequência dos eventos, os pacientes acabam por deixar de tomar os medicamentos, resultado que converge com esse estudo, causando falhas na ação dos mesmos ${ }^{(22)}$. Assim, destaca-se a importância das informações, esclarecimentos e estabelecimento de estratégias que minimizem os efeitos colaterais dos ARV no início e durante o tratamento, os quais podem ser realizados durante o acompanhamento no serviço de saúde ${ }^{(12)}$.

Para os pacientes com medicação no horário do trabalho, existem duas problemáticas, uma está relacionada às medicações, onde elas podem afetar as condições psicológicas, dificultando o desenvolvimento do trabalho ${ }^{(6)}$. A outra refere-se aos pacientes que não realizaram a revelação de seu diagnóstico no local onde estão empregados, por esse motivo, muitas vezes, deixam de fazer o uso da medicação pelo medo da descoberta e discriminação dos colegas ${ }^{(9)}$, uma vez que alguns ARV necessitam de cuidados especiais, como por exemplo, mantê-los sob refrigeração. Ao utilizar um refrigerador de uso comum, a possibilidade de revelar o diagnóstico às pessoas do trabalho aumenta, desencadeando um impacto negativo na realização da $\operatorname{TARV}^{(23)}$. 


\section{CONSIDERAÇÕES FINAIS}

A partir da classificação da adesão à TARV foi possível identificar que o índice de adultos não aderentes está acima das taxas médias de outras populações já analisadas. Porém, destaca-se que a definição da adesão é variável, e pode sofrer influência conforme a medida utilizada para defini-la.

Dessa forma, observa-se a necessidade de ampliar a discussão em torno da temática. visto que os diferentes motivos que levaram as pessoas a não ingerirem doses dos medicamentos em algum momento da TARV, propõem-se a elaboração de estratégias que atendam às necessidades individuais. Acredita-se que, por meio de ações de educação em saúde que abranjam a (re)construção dos hábitos dessas pessoas, será possível ampliar a cooperação do paciente a fim de melhorar a adesão à terapia e reduzir as complicações que podem acometer o indivíduo em situação de supressão imunitária.

\section{REFERÊNCIAS}

1. Padoin SMM, Paula CC, Züge SS, Primeira MR, Santos EEP, Tolentino LC. Fatores associados a não adesão ao tratamento antirretroviral em adultos acima de 50 anos que tem HIV/AIDS. DST, j. bras. doencas sex. transm. [Internet] 2011;23(4) [acesso em $01 \mathrm{dez} 2012$ ]. Disponível: http://www.dst.uff.br/revista23-42011/9. Fatores $\% 20$ associados $\% 20 \mathrm{a} \% 20$ nao $\% 20$ adesao $\% 20$ ao\%20tratamento.pdf

2. Brasil. Lei n.9.313, de 13 de novembro de 1996. Dispõe sobre a distribuição gratuita de medicamentos aos portadores do HIV e doentes de AIDS. Diário Oficial da República Federativa do Brasil. Brasília, 13 de novembro de 1996. [Internet] [acesso em $01 \mathrm{dez} 2012$ ]. Disponível: http://www.aids.gov.br/sites/default/files/ anexo_2_1_002.pdf

3. Polejack L, Seidl EMF. Monitoramento e avaliação da adesão ao tratamento antirretroviral para HIV/AIDS: desafios e possibilidades. Ciênc. saúde colet. [Internet] 2010;15(Suppl1) [acesso em $01 \mathrm{dez} 2012$ ]. Disponível: http://dx.doi.org/10.1590/S1413-81232010000700029

4. Silva ALCN, Waidman MAP, Marcon SS. Adesão e não adesão à terapia anti-retroviral: as duas faces de uma mesma vivência. Rev. bras. enferm. [Internet] 2009; 62(2) [acesso em $01 \mathrm{dez} 2012$ ]. Disponível: http://dx.doi. org/10.1590/S0034-71672009000200007

5. Padoin SMM, Machiesqui SR, Paula CC, Tronco CS, De Marchi MC. Cotidiano terapêutico de adultos portadores da síndrome da imunodeficiência adquirida. Rev. Enferm. UERJ. [Internet] 2010;18(3):389-93 [acesso em 01 dez 2012]. Disponível: http://www.facenf. uerj.br/v18n3/v18n3a09.pdf

6. Seidl EMF, Melchíades A, Farias V, Brito A. Pessoas vivendo com HIV/AIDS: variáveis associadas à adesão ao tratamento anti-retroviral. Cad. Saúde Pública. [Internet] 2007;23(10): 2305-16 [acesso em $01 \mathrm{dez}$ 2012]. Disponível: http://dx.doi.org/10.1590/S0102311X2007001000006

7. Pinto ACS, Pinheiro PNC, Vieira NFC, Alves MDS. Compreensão da pandemia de AIDS nos últimos 25 anos. DST, j. bras. doencas sex. transm. [Internet] 2007;19(1):45-50 [acesso em 01 dez 2012]. Disponível: http://www.dst.uff.br//revista19-1-2007/7.pdf

8. Ministério da Saúde (BR). Secretaria de Vigilância em Saúde. Departamento Nacional de DST e Aids. Boletim Epidemiológico Aids/DST; 2012.

9. Pereira LB, Albuquerque JR, Santos JM, Lima FLA, Saldanha AAW. Fatores sociodemográficos e clínicos associados à TARV e a contagem T-CD4. Revista Brasileira de Ciência Saúde. [Internet] 2012;16(2):149-60 [acesso em 01 dez 2012]. Disponível: http://periodicos.ufpb.br/ojs/index.php/rbcs/article/ view/11544/7321

10. Silva CM, Lopes FMVM, Vargens OMC. A vulnerabilidade da mulher idosa em relação à Aids. Rev. Gaúcha Enferm. [Internet] 2010;31(3):450-7 [acesso em 01 dez 2012]. Disponível: http://dx.doi.org/10.1590/ S1983-14472010000300007

11. Silva CM, Vargens OMC. A percepção de mulheres quanto à vulnerabilidade feminina para contrair DST/ HIV. Rev Esc Enferm USP. [Internet] 2009;43(2):4016 [acesso em $01 \mathrm{dez} 2012$ ]. Disponível: http://dx.doi. org/10.1590/S0080-62342009000200020

12. Almeida EL, Araujo GBS, Santos VA, Bustorff LACV, Pereira AVL, Dias MD. Adesão dos portadores do hiv/aids ao tratamento: fatores intervenientes. Rev. Min. Enferm. [Internet] 2011;15(2):208-16 [acesso em $01 \mathrm{dez} 2012$ ]. Disponível: http://www.enf.ufmg.br/site_novo/modules/ mastop_publish/files/files_4e662b $005 a 6 b 3 . p d f$

13. Santos FB, Seidl EMF. Caracterização de pessoas com HIV/AIDS em abandono do tratamento antirretroviral e a busca consentida de casos. Brasilia med. [Internet] 2011;48(3) [acesso em 01 dez 2012]. Disponível: http:// www.ambr.org.br/portal/arquivos/abandono_do_ tratamento_de_aids.pdf 
14. Schilkowsky LB, Portela MC, Sa MC. Fatores associados ao abandono de acompanhamento ambulatorial em um serviço de assistência especializada em HIV/aids na cidade do Rio de Janeiro, RJ. Rev. bras. epidemiol. [Internet] 2011;14(2):187-97 [acesso em $01 \mathrm{dez}$ 2012]. Disponível: http://dx.doi.org/10.1590/S1415790X2011000200001.

15. Aboim S. Risco e prevenção do HIV/Aids: uma perspectiva biográfica sobre os comportamentos sexuais em Portugal. Ciênc. saúde colet. [Internet] 2012;17(1):99-112 [acesso em 01 dez 2012]. Disponível: http://dx.doi.org/10.1590/S141381232012000100013 16. Helena ETS, Mafra ML, Simes $\mathrm{M}$. Fatores associados à sobrevida de pessoas vivendo com aids no Município de Blumenau, Estado de Santa Catarina, Brasil, 1997-2004. Epidemiol. Serv. Saude. [Internet] 2009;18(1):45-53 [acesso em $01 \mathrm{dez} 2012$ ]. Disponível: http://scielo.iec.pa.gov.br/pdf/ess/v18n1/v18n1a05.pdf

17. Ilias M, Carandina L, Marin MJS. Adesão à terapia antirretroviral de portadores do vírus da imunodeficiência humana atendidos em um ambulatório da cidade de Marília, São Paulo. RBSP. [Internet] 2011;35(2):47184 [acesso em $01 \mathrm{dez} 2012$ ]. Disponível: http://files. bvs.br/upload/S/0100-0233/2011/v35n2/a2470.pdf

18. Melchior R, Nemes MIB, Alencar TMD, Buchalla CM. Desafios da adesão ao tratamento de pessoas vivendo com HIV/AIDS no Brasil. Rev. Saúde Públ. [Internet] 2007;41(Suppl. 2):87-93 [acesso em $01 \mathrm{dez}$ 2012]. Disponível: http://dx.doi.org/10.1590/S003489102007000900014

19. Blatt CR, Citadin CB, Souza FG, Mello RS, Galato D. Avaliação da adesão aos anti-retrovirais em um município no Sul do Brasil. Rev. Soc. Bras. Med. Trop. [Internet] 2009;42(2):131-6 [acesso em $01 \mathrm{dez}$ 2012]. Disponível: http://dx.doi.org/10.1590/S003786822009000200007 .

20. Padoin SMM, Paula CC, Zuge SS, Primeira MR, Santos EEP, Tolentino LC. Fatores Associados à não adesão ao tratamento antirretroviral em adultos acima de 50 anos que têm HIV/AIDS. DST, j. bras. doencas sex. transm. [Internet] 2011;23(4):194-7 [acesso em $01 \mathrm{dez} 2012$ ]. Disponível: http://www.dst.uff.br/revista23-4-2011/9. Fatores $\% 20$ associados $\% 20 a \% 20$ nao $\% 20$ adesao $\% 20$ ao\%20tratamento.pdf

21. Rego SRM, Oliveira CFA, Rego DMS, Júnior RFS, Silva VB. Estudo do autorrelato de adesão e uso problemático de álcool em uma população de indivíduos com AIDS em uso de HAART. J. bras. psiquiatr. [Internet] 201;60(1):46-9 [acesso em 01 dez 2012]. Disponível: http://www.scielo.br/pdf/jbpsiq/v60n1/v60n1a09.pdf
22. Moriel P, Carnevale RC, Costa CGR, Braz NC, Santos CZ, Baleiro LS, et al. Efeitos das intervenções farmacêuticas em pacientes hiv positivos: influência nos problemas farmacoterapêuticos, parâmetros clínicos e economia. R. Bras. Farm. Hosp. Serv. Saúde São Paulo. [Internet] 2011;2(3):5-10 [acesso em 01 dez 2012]. Disponível: http://www.sbrafh.org.br/rbfhss/public/ artigos/RBFHSSV2N3\%20artigo01.pdf

23. Colombrini MRC, Lopes MHBM, Figueiredo RM. Adesão à terapia antirretroviral para HIV/AIDS. Rev Esc Enferm USP. [Internet] 2006;40(4): 576-81[acesso em $01 \mathrm{dez}$ 2012]. Disponível: http://dx.doi.org/10.1590/ S0080-62342006000400018. 